\title{
DIVERGENT AND CONDITIONALLY CONVERGENT SERIES WHOSE PRODUCT IS ABSOLUTELY CONVERGENT*
}

BY

\author{
FLORIAN CAJORI
}

\section{§1. Introduction.}

It has been shown by ABEL that, if the product :

$$
\sum_{n=0}^{n=\infty}\left(u_{0} v_{n}+u_{1} v_{n-1}+\cdots+u_{n} v_{0}\right),
$$

of two conditionally convergent series :

$$
\sum_{n=0}^{n=\infty} u_{n}, \quad \sum_{n=0}^{n=\infty} v_{n},
$$

is convergent, it converges to the product of their sums. Tests of the convergence of the product of conditionally convergent series have been worked out by A. Pringsheim, $\dagger$ A. Voss, $\ddagger$ and myself. $\S$ There exist certain conditionallyconvergent series which yield a convergent result when they are raised to a certain positive integral power, but which yield a divergent result when they are raised to a higher power. Thus,

$$
\sum_{n=1}^{n=\infty}(-1)^{n+1} \frac{1}{n^{r}}
$$

where $r=7 / 9$, is a conditionally convergent series whose fourth power is convergent, but whose fifth power is divergent.\| These instances of conditionally

* Presented to the Society A pril 28, 1900. Received for publication April 28, 1900.

† Mathematische Annalen, vol. 21 (1883), p. 327 ; vol. 26 (1886), p. 157.

$\ddagger$ Mathematische Annalen, vol. 24 (1884), p. 42.

\$ A merican Journal of Mathematics, vol. 15 (1893), p. 339 ; vol. 18 (1896), p. 195 ; Bulletin of the American Mathematical Society, (2) vol. 1 (1895), p. 180.

$\|$ This may be a convenient place to point out a slight and obvious extension of the results which I have published in the American Journal of Mathematics, vol. 18, p. 201. It was proved there that the conditionally convergent series :

$$
\sum_{n=1}^{n=\infty}(-1)^{n+1} \frac{1}{n^{r}}
$$

$$
(0<r \equiv 1),
$$

when raised by Cauchy's multiplication rule to a positive integral power $q$, is convergent whenever $(q-1) / q<r$; but the power of the series is divergent, if $(q-1) / q>r$. The case for which $(q-1) / q=r$ was left untouched, but an examination of the formulæ appearing in that article readily yields the result that in this case the series is divergent. Hence the condition for divergeucy should be written $(q-1) / q \geqq r$. 
convergent series, yielding powers of the series which converge less rapidly the higher the power and which for a sufficiently high power yield a divergent result, suggest the following questions: Does the product of two conditionally convergent series never converge more rapidly than do one or both of the factorseries? Can the product of two conditionally convergent series or of a conditionally convergent and a divergent series in no case be absolutely convergent?

The first doubt of the correctness of a negative reply arose in connection with the conditionally convergent series :

$$
\sum_{n=1}^{n=\infty} \frac{(-1)^{n+1}}{n}
$$

This yields convergent results, no matter to how high a positive integral power it may be raised.* If the series is squared, the constituents, $1_{\prime}^{\prime}(n-r)(r+1)$, which go to make up the $n$th term of the product, namely,

$$
\sum_{r=0}^{r=n-1} \frac{1}{n-r} \frac{1}{r+1}
$$

are all positive or all negative for that term. Hence the numerical value of the $n$th term is the sum of the numerical values of all the products $1 /(n-r)(r+1)$. If the square of the series is indicated by

$$
\sum_{n=1}^{n=\infty} a_{n}
$$

then all the constituents, $a_{n-r} \cdot 1 /(r+1)$, which enter into the composition of the $n$th term in the cube of the series have all like signs in that term. Similarly for higher powers. Now it has been shown that all the positive integral powers of the conditionally convergent series :

$$
\sum_{n=1}^{n=\infty} \frac{(-1)^{n+1}}{n}
$$

continue to converge, even though each term of a given power of the series is numerically the sum of the numerical values of its constituents, and it is evident that, if the constituents of each term were partly positive and partly negative, each term would be numerically smaller. Can the signs of the terms of two conditionally convergent series and the numerical values of its terms be so chosen that the product of the two series is absolutely convergent?

In an important article on the multiplication of conditionally convergent series $\dagger$ A. Pringsheim states that he knows no a priori reason why there might not be special cases in which such a product is absolutely convergent. "Aller-

* American Journal of Mathematics, vol. 18, p. 204.

† Mathematische Annalen, vol. 21, p. 332. 
dings," he continues, "war es mir bisher nicht möglich an irgend einem bestimmten derartigen Falle die unbedingte Convergenz zu constatiren, und es dürfte dies - wenn es überhaupt solche Fälle giebt, was ja immerhin als fraglich erscheinen muss - wegen der zusammengesetzten Beschaffenheit der Reihenglieder $w_{\nu}$ eben nur sehr schwer gelingen."

\section{§2. Two typical examples.}

We have succeeded in devising special cases in which an absolutely convergent series is obtained as the result of multiplying two conditionally convergent series together, or one conditionally convergent series by a divergent series. The series obtained by removing the parentheses from the series :

$$
\begin{aligned}
& S_{1} \equiv \sum_{p=0}^{p=\infty}\left(\frac{1}{4 p+1}-\frac{1}{4 p+4}+\frac{1}{4 p+1}-\frac{1}{4 p+4}\right), \\
& S_{2} \equiv \sum_{p=0}^{p=\infty}\left(\frac{1}{4 p+1}+\frac{1}{4 p+4}-\frac{1}{4 p+1}-\frac{1}{4 p+4}\right),
\end{aligned}
$$

are both conditionally convergent. Let the four terms obtained in either series, or the terms in a similar series, for any one positive integral value of $p$, be called a "set" of terms. The $4 n$-th term of the product of $S_{1}$ and $S_{2}$ is

$$
\begin{aligned}
\sum_{p=0}^{p=n-1}\left(-\frac{1}{4 n-4 p} \frac{1}{4 p+1}+\frac{1}{4 n-4 p-3} \frac{1}{4 p+4}\right. & \\
& \left.\quad+\frac{1}{4 n-4 p} \frac{1}{4 p+1}-\frac{1}{4 n-4 p-3} \frac{1}{4 p+4}\right) .
\end{aligned}
$$

Wc see that the sum of each set of constituents for the $4 n$-th term is identically zero ; therefore the entire $4 n$-th term is identically zero.

The $(4 n+1)$-th term of the product series is as follows:

$$
\begin{aligned}
\sum_{p=0}^{p=n^{\prime}}\left(\frac{1}{4 n-4 p+1} \frac{1}{4 p+1}-\frac{1}{4 n-4 p}\right. & \frac{1}{4 p+4} \\
& \left.\quad-\frac{1}{4 n-4 p-3} \frac{1}{4 p+1}+\frac{1}{4 n-4 p} \frac{1}{4 p+4}\right) \\
+\sum_{p=0}^{p=n^{\prime}}\left(\frac{1}{4 n-4 p+1} \frac{1}{4 p+1}+\right. & \frac{1}{4 n-4 p} \frac{1}{4 p+4} \\
& \left.-\frac{1}{4 n-4 p-3} \frac{1}{4 p+1}-\frac{1}{4 n-4 p} \frac{1}{4 p+4}\right)-a,
\end{aligned}
$$

where $n^{\prime}=\frac{1}{2} n-1$ or $\frac{1}{2}(n-1)$, according as $n$ is even or odd. When $n$ is odd,

$$
a=\frac{1}{2 n+2} \frac{1}{2 n+2}-\frac{1}{2 n-1} \frac{1}{2 n-1}-\frac{1}{2 n+2} \frac{1}{2 n+2}=-\frac{1}{2 n-1} \frac{1}{2 n-1} ;
$$


and when $n$ is even,

$$
a=-\frac{1}{2 n+1} \frac{1}{2 n+1}
$$

In the first summation we are adding $4 n^{\prime}+4$ constituents from one end of the column or series yielding the $(4 n+1)$-th term of the product; in the second summation we are adding $4 n^{\prime}+4$ constituents from the other end. In the middle of the series the sets of constituents will overlap, if $n$ is odd, and will not touch if $n$ is even. When $n$ is odd, then a represents the sum of the constituents which have been counted twice; when $n$ is even, then $a$ stands for the negative value of the constituent not counted at all. Each of these constituents is of the degree -2 with respect to $n$ and as their number is finite, their sum cannot be of a higher degree with respect to $n$ than the degree -2 .

The $(4 n+1)$-th term, simplified, is

$$
2 \sum_{p=0}^{p=n^{\prime}}\left(\frac{1}{4 p+1} \frac{-4}{(4 n-4 p+1)(4 n-4 p-3)}\right)-a .
$$

For any positive integral value of $n$ the fraction $4 /(4 n-4 p+1)(4 n-4 p-3)$ has its maximum value when $p=n^{\prime}$. Writing $n^{\prime}$ for $p$, we obtain a fraction which is less than $1 / n^{2}$. Hence for any value of $p$,

$$
\frac{1}{4 p+1} \frac{4}{(4 n-4 p+1)(4 n-4 p-3)}<\frac{1}{4 p+1} \frac{1}{n^{2}} .
$$

It is easy to see that

$$
\sum_{n=1}^{2 n} \frac{1}{n}<a \log (2 n),
$$

where $a$ is a constant greater than $3 / 2 \log 2$. Hence

and

$$
\sum_{p=0}^{p=n^{\prime}} \frac{1}{4 p+1}<a \log (2 n)
$$

$$
\sum_{p=0}^{p=n^{\prime}} \frac{1}{4 p} \frac{4}{4} \frac{4}{(4 n-4 p+1)(4 n-4 p-3)}<\sum_{p=0}^{p=n^{\prime}} \frac{1}{4 p+1} \frac{1}{n^{2}}<\frac{a \log (2 n)}{n^{2}} .
$$

But a number $m$ can be found, such that, when $n>m$, we have

$$
\frac{a \log (2 n)}{n^{2}}<\frac{1}{n(\log n)^{\theta}}
$$

where $\theta>1$. Therefore, for $n>m$, the $(4 n+1)$-th term is numerically less than the $n$th term of a series known to be absolutely convergent. 
The $(4 n+2)$-th term of the product-series is

$$
\begin{aligned}
\sum_{p=0}^{p=n^{\prime}}\left(-\frac{1}{4 n-4 p+4} \frac{1}{4 p+1}\right. & +\frac{1}{4 n-4 p+1} \frac{1}{4 p+4} \\
& \left.\quad+\frac{1}{4 n-4 p} \frac{1}{4 p+1}-\frac{1}{4 n-4 p-3} \frac{1}{4 p+4}\right) \\
+\sum_{p=0}^{p=n^{\prime}}\left(+\frac{1}{4 n-4 p+4} \frac{1}{4 p+1}-\frac{1}{4 n-4 p+1 \frac{1}{4 p+4}}\right. & \left.-\frac{1}{4 n-4 p} \frac{1}{4 p+1}+\frac{1}{4 n-4 p-3} \frac{1}{4 p+4}\right)-a,
\end{aligned}
$$

where $n^{\prime}$ is defined as before and where

$$
a=\frac{1}{2 n+2} \frac{1}{2 n-1}-\frac{1}{2 n-1} \frac{1}{2 n+2}=0,
$$

when $n$ is odd, and

$$
a=+\frac{1}{2 n+4} \frac{1}{2 n+1}-\frac{1}{2 n+1} \frac{1}{2 n+4}=0,
$$

when $n$ is even. We see that the entire $(4 n+2)$-th term is identically equal to zero.

The $(4 n+3)$-th term of the product-series is

$$
\begin{gathered}
\sum_{p=0}^{p=n^{\prime}}\left(+\frac{1}{4 n-4 p+1} \frac{1}{4 p+1}-\frac{1}{4 n-4 p+4} \frac{1}{4 p+4}\right. \\
\left.-\frac{1}{4 n-4 p+1} \frac{1}{4 p+1}+\frac{1}{4 n-4 p} \frac{1}{4 p+4}\right) \\
+\sum_{p=0}^{p=n^{\prime}}\left(-\frac{1}{4 n-4 p+1} \frac{1}{4 p+1}-\frac{1}{4 n-4 p+4} \frac{1}{4 p+4}\right. \\
\left.+\frac{1}{4 n-4 p+1} \frac{1}{4 p+1}+\frac{1}{4 n-4 p} \frac{1}{4 p+4}\right)-a \\
=2 \sum_{p=0}^{p=n^{\prime}}\left(\frac{1}{4 p+4} \frac{4}{(4 n-4 p+4)(4 n-4 p)}\right)-a,
\end{gathered}
$$

where

when $n$ is odd, and

$$
a=\frac{1}{2 n+2} \frac{1}{2 n+2}
$$

$$
a=-\frac{1}{2 n+1} \frac{1}{2 n+1}+\frac{1}{2 n+4} \frac{1}{2 n+4}+\frac{1}{2 n+1} \frac{1}{2 n+1},
$$


when $n$ is even. The reasoning which we applied to the $(4 n+1)$-th term will show that this term, too, when $n>m$, is numerically less than $1 / n(\log n)^{\theta}$, $\theta>1$.

We have now shown that the $4 n$-th term and the three immediately succeeding terms are either identically zero, or, for $n>m$, numerically less than $1 / n(\log n)^{\theta}$. Hence the product of the series $S_{1}$ and $S_{2}$ converges absolutely.

Again, take the conditionally convergent series, obtained by dropping the parentheses in the series:

$$
T_{1} \equiv \sum_{p=0}^{p=\infty}\left(\frac{1}{6 p+1}-\frac{1}{6 p+2}+\frac{1}{6 p+3}-\frac{1}{6 p+1}+\frac{1}{6 p+2}-\frac{1}{6 p+3}\right)
$$

and the divergent series, obtained by dropping the parentheses in the series:

$$
T_{2} \equiv \sum_{p=0}^{p=\infty}\left(\frac{1}{6 p+1}+\frac{1}{6 p+2}-\frac{1}{6 p+3}+\frac{1}{6 p+1}+\frac{1}{6 p+2}-\frac{1}{6 p+3}\right) .
$$

The $6 n$-th term of their product, viz.,

$$
\begin{aligned}
\sum_{p=0}^{p=n-1}(- & \frac{1}{6 n-6 p+3} \frac{1}{6 p+1}+\frac{1}{6 n-6 p+2} \frac{1}{6 p+2}+\frac{1}{6 n-6 p+1} \frac{1}{6 p+3} \\
& \left.+\frac{1}{6 n-6 p+3} \frac{1}{6 p+1}-\frac{1}{6 n-6 p+2} \frac{1}{6 p+2}-\frac{1}{6 n-6 p+1} \frac{1}{6 p+3}\right)
\end{aligned}
$$

is seen to be identically zero.

The $(6 n+1)$-th term of the product of $T_{1}$ and $T_{2}$ is

$$
\begin{gathered}
\sum_{p=0}^{p=n^{\prime}}\left(+\frac{1}{6 n-6 p+1} \frac{1}{6 p+1}-\frac{1}{6 n-6 p-3} \frac{1}{6 p+2}-\frac{1}{6 n-6 p-4} \frac{1}{6 p+3}\right. \\
\left.-\frac{1}{6 n-6 p-5} \frac{1}{6 p+1}+\frac{1}{6 n-6} \frac{1}{6 p-3}+\frac{1}{6 n+6}+\frac{1}{6 p+3}\right) \\
+\sum_{p=0}^{p=n^{\prime}}\left(+\frac{1}{6 n-6 p+1} \frac{1}{6 p+1}+\frac{1}{6 n-6 p-3} \frac{1}{6 p+2}+\frac{1}{6 n-6 p-4} \frac{1}{6 p+3}\right. \\
\left.-\frac{1}{6 n-6 p-5} \frac{1}{6 p+1}-\frac{1}{6 n-6 p-3} \frac{1}{6 p+2}-\frac{1}{6 n-6 p-4} \frac{1}{6 p+3}\right)-a \\
=2 \sum_{p=0}^{p=n^{\prime}}\left(\frac{1}{6 p+1} \frac{-6}{(6 n-6 p+1)(6 n-6 p-5)}\right)-a,
\end{gathered}
$$

where $n^{\prime}$ is defined as before and where 


$$
\begin{gathered}
a=-\frac{1}{3 n} \frac{1}{3 n-1}-\frac{1}{3 n-1} \frac{1}{3 n}-\frac{1}{3 n-2} \frac{1}{3 n-2}+\frac{1}{3 n} \frac{1}{3 n-1}+\frac{1}{3 n-1} \frac{1}{3 n} \\
=-\frac{1}{3 n-2} \frac{1}{3 n-2},
\end{gathered}
$$

when $n$ is odd, and $a=-1 /(3 n+1)(3 n+1)$, when $n$ is even. By repeating the reasoning used in previous cases we find that this term, for $n>m$, is numerically less than $1 / n(\log n)^{\theta}, \theta>1$.

The $(6 n+3)$-th, $(6 n+4)$-th, and $(6 n+5)$-th terms in the product of $T_{1}$ and $T_{2}$, when subjected to this course of reasoning, all yield the same conclusion. The $(6 n+2)$-th term is identically zero. Hence the product-series is absolutely convergent.

\section{§3. The general method.}

The method by which the above factor-series were constructed is simple. The harmonic series was modified by adopting some simple law of succession of signs. To render the series conditionally convergent, make the number of + terms in a set equal to the number of - terms. If these numbers are unequal, then the series is divergent. Then add suitable finite numbers to the denominators of some of the terms in the set, so that, when the product of the two series is formed, the constituents of any set of the $n$th term in the product, when added, yield a fractional sum having denominators whose degree in $n$ is higher by two than the degree of the numerators. We were able to prove the absolute convergence from the fact that in each complete set of constitutents of the $n$th term in the product-series there are as many positive constituents 'as there are negative ones. To secure this even distribution of signs among the constituents for the sets in every term of the product, it is necessary that in one of the factorseries the number of + terms in any set be equal to the number of - terms in that set. This condition is not sufficient, because among the terms in the product some might have a preponderance of + constituents and others a preponderance of - constituents. This happens, for instance, when we multiply the series ++--++-- etc. by itself.

In the proper selection of signs the following rule, which we give without proof, may be of service. Let $a$, denoting the number of terms in each set of the series $\sum u_{m}$, be an even number and equal to $2 c$; also let the first $c$ terms in each set be + , the remaining terms - Let the number of terms in each set of the second series, $\sum v_{m}$, be $b$. Then, in order to secure as many + constituents in each set of a term of the product of the two series $\sum u_{m}$ and $\sum v_{m}$, as there are negative constituents, it is sufficient that $d$ should be a factor of $c$, where $d$ is the smallest value, numerically, satisfying the congruence $b \equiv d(\bmod a)$. 
In explaining the further process of constructing our series we shall start out with a particular selection of signs, but the process is the same as for any other selection, similarly made. Let $a=4, b=3$, then $c=2$ and $d=-1$, a factor of $c$. Accordingly, the signs of terms in each set of the first series are ++-- . Let the signs of the $b$ terms in each set of $\sum v_{m}$ be ++- . We have now the two factor-series obtained by dropping the parentheses from the series on the right hand sides of the formulas:

and

$$
\sum u_{m} \equiv \sum_{p=1}^{p=\infty}\left(\frac{1}{4 p_{1}+a_{1}}+\frac{1}{4 p+1+a_{2}}-\frac{1}{4 p+2+a_{3}}-\frac{1}{4 p+3+a_{4}}\right)
$$

$$
\sum v_{m} \equiv \sum_{p=1}^{p=\infty}\left(\frac{1}{3 p+\beta_{1}}+\frac{1}{3 p+1+\beta_{2}}-\frac{1}{3 p+2+\beta_{3}}\right)
$$

in which the Greek letters are quantities to be chosen, if possible, so that the product of the two series shall be absolutely convergent.

For brevity, we shall, in the series $\sum u_{m}$, represent by $\left\{u_{a p}+a\right\}$ a fraction $1 /(a p+a)$, and, similarly, in the series $\sum v_{m}$, by $\left\{v_{b p}+\beta\right\}$ a fraction $1 /(b p+\beta)$. Accordingly we have, dropping the parentheses as before,

$$
\sum u_{m} \equiv \sum_{p=1}^{p=\infty}\left(\left\{u_{4 p}+a_{1}\right\}+\left\{u_{4 p}+1+a_{2}\right\}-\left\{u_{4 p}+2+a_{3}\right\}-\left\{u_{4 p}+3+a_{4}\right\}\right)
$$

and

$$
\sum v_{m} \equiv \sum_{p=1}^{p=\infty}\left(\left\{v_{3 p}+\beta_{1}\right\}+\left\{v_{3 p}+1+\beta_{2}\right\}-\left\{v_{3 p}+2+\beta_{3}\right\}\right) \text {. }
$$

In the product of $\sum u_{m}$ and $\sum v_{m}$, a set will consist of 12 constituents. The first set in the $(12 n)$-th term will be (since $\left\{v_{12}-9+\beta_{1}\right\} \equiv\left\{v_{3}+\beta_{1}\right\}$, and similarly in other cases)

$$
\left\{\begin{array}{l}
-\left\{u_{12 n}+3+a_{4}^{\prime \prime}\right\}\left\{v_{12}-9+\beta_{1}\right\}-\left\{u_{12 n}+2+a_{3}^{\prime \prime}\right\}\left\{v_{12}-8+\beta_{2}\right\} \\
-\left\{u_{12 n}+1+a_{2}^{\prime \prime}\right\}\left\{v_{12}-7+\beta_{3}\right\}+\left\{u_{12 n}+a_{1}^{\prime \prime}\right\}\left\{v_{12}-6+\beta_{1}^{\prime}\right\} \\
-\left\{u_{12 n}-1+a_{4}^{\prime}\right\}\left\{v_{12}-5+\beta_{2}^{\prime}\right\}+\left\{u_{12 n}-2+a_{3}^{\prime}\right\}\left\{v_{12}-4+\beta_{3}^{\prime}\right\} \\
+\left\{u_{12 n}-3+a_{2}^{\prime}\right\}\left\{v_{12}-3+\beta_{1}^{\prime \prime}\right\}+\left\{u_{12 n}-4+a_{1}^{\prime}\right\}\left\{v_{12}-2+\beta_{2}^{\prime \prime}\right\} \\
+\left\{u_{12 n}-5+a_{4}\right\}\left\{v_{12}-1+\beta_{3}^{\prime \prime}\right\}-\left\{u_{12 n}-6+a_{3}\right\}\left\{v_{12}+\beta_{1}^{\prime \prime \prime}\right\} \\
+\left\{u_{12 n}-7+a_{2}\right\}\left\{v_{12}+1+\beta_{2}^{\prime \prime \prime}\right\}-\left\{u_{12 n}-8+a_{1}\right\}\left\{v_{12}+2+\beta_{3}^{\prime \prime \prime}\right\} .
\end{array}\right.
$$

Here each of the quantities $\beta_{1}, \beta_{2}, \beta_{3}$ occurs four times. We have broadened our assumptions by attaching a single accent to each letter the first time it is repeated and a double and triple accent the second and third time it is repeated. Accordingly $\beta_{1}, \beta_{1}^{\prime}, \beta_{1}^{\prime \prime}, \beta_{1}^{\prime \prime}, \beta_{2}, \beta_{2}^{\prime}, \beta_{2}^{\prime \prime}, \beta_{2}^{\prime \prime \prime}, \beta_{3}, \beta_{3}^{\prime}, \beta_{3}^{\prime \prime}, \beta_{3}^{\prime \prime \prime}$ may in general represent each a different number. Similarly, the letters $a_{1}, a_{2}, a_{3}, a_{4}$ receive a single accent the first time they are repeated in a set of constituents and a double accent the second time they are repeated. 
We shall limit ourselves to the case where, in any term of the product series, each set, when multiplied by $n$, yields a product that converges toward zero when $n=\infty$. Accordingly, if the set I be multiplied by $n$, we get, for $n=\infty$ (supposing the Greek letters to be positive constants, or variables which approach positive finite limits), the first of the two following equations :

$$
\left\{\begin{array}{l}
-\left\{v_{12}-9+\beta_{1}\right\}-\left\{v_{12}-8+\beta_{2}\right\}-\left\{v_{12}-7+\beta_{3}\right\}+\left\{v_{12}-6+\beta_{1}^{\prime}\right\} \\
-\left\{v_{12}-5+\beta_{2}^{\prime}\right\}+\left\{v_{12}-4+\beta_{3}^{\prime}\right\}+\left\{v_{12}-3+\beta_{1}^{\prime \prime}\right\}+\left\{v_{12}-2+\beta_{2}^{\prime \prime}\right\} \\
+\left\{v_{12}-1+\beta_{3}^{\prime \prime}\right\}-\left\{v_{12}+\beta_{1}^{\prime \prime}\right\}+\left\{v_{12}+1+\beta_{2}^{\prime \prime}\right\}-\left\{v_{12}+2+\beta_{3}^{\prime \prime}\right\}=0, \\
+\left\{v_{12}-9+\beta_{1}\right\}-\left\{v_{12}-8+\beta_{2}\right\}+\left\{v_{12}-7+\beta_{3}\right\}+\left\{v_{12}-6+\beta_{1}^{\prime}\right\} \\
+\left\{v_{12}-5+\beta_{2}^{\prime}\right\}+\left\{v_{12}-4+\beta_{3}^{\prime}\right\}-\left\{v_{12}-3+\beta_{1}^{\prime \prime}\right\}+\left\{v_{12}-2+\beta_{2}^{\prime \prime}\right\} \\
-\left\{v_{12}-1+\beta_{3}^{\prime \prime}\right\}-\left\{v_{12}+\beta_{1}^{\prime \prime \prime}\right\}-\left\{v_{12}+1+\beta_{2}^{\prime \prime}\right\}-\left\{v_{12}+2+\beta_{3}^{\prime \prime}\right\}=0 .
\end{array}\right.
$$

The second equation in II is got from the $(12 n+1)$-th term by the same procedure. All the even terms between the $(12 n)$-th and the $(12 n+12)$-th yield the same equation as the $(12 n)$-th term; all the odd terms yield the same equation as the $(12 n+1)$-th term.

The last set in the $(12 n)$-th term of the product is

$$
\left\{\begin{array}{l}
-\left\{v_{12 n}+2+\beta_{3}^{\prime \prime \prime}\right\}\left\{u_{12}-8+a_{1}\right\}+\left\{v_{12 n}+1+\beta_{2}^{\prime \prime}\right\}\left\{u_{12}-7+a_{2}\right\} \\
-\left\{v_{12 n}-1+\beta_{1}^{\prime \prime \prime}\right\}\left\{u_{12}-6+a_{3}\right\}+\left\{v_{12 n}-1+\beta_{3}^{\prime \prime}\right\}\left\{u_{12}-5+a_{4}\right\} \\
+\left\{v_{12 n}-2+\beta_{2}^{\prime \prime}\right\}\left\{u_{12}-4+a_{1}^{\prime}\right\}+\left\{v_{12 n}-3+\beta_{1}^{\prime \prime}\right\}\left\{u_{12}-3+a_{2}^{\prime}\right\} \\
+\left\{v_{12 n}-4+\beta_{3}^{\prime}\right\}\left\{u_{12}-2+a_{3}^{\prime}\right\}-\left\{v_{12 n}-5+\beta_{2}^{\prime}\right\}\left\{u_{12}-1+a_{4}^{\prime}\right\} \\
+\left\{v_{12 n}-6+\beta_{1}^{\prime}\right\}\left\{u_{12}-a_{1}^{\prime \prime}\right\}-\left\{v_{12 n}-7+\beta_{3}\right\}\left\{u_{12}+1+a_{2}^{\prime \prime}\right\} \\
-\left\{v_{12 n}-8+\beta_{2}\right\}\left\{u_{12}+2+a_{3}^{\prime \prime}\right\}-\left\{v_{12 n}-9+\beta_{1}\right\}\left\{u_{12}+3+a_{4}^{\prime \prime}\right\} .
\end{array}\right.
$$

Assuming set III, when multiplied by $n$, to yield a product which converges toward zero, when $n=\infty$, we get the first of the following three equations :

(IV)

$$
\left\{\begin{array}{l}
-\left\{u_{12}-8+a_{1}\right\}+\left\{u_{12}-7+a_{2}\right\}-\left\{u_{12}-6+a_{3}\right\}+\left\{u_{12}-5+a_{4}\right\} \\
+\left\{u_{12}-4+a_{1}^{\prime}\right\}+\left\{u_{12}-3+a_{2}^{\prime}\right\}+\left\{u_{12}-2+a_{3}^{\prime}\right\}-\left\{u_{12}-1+a_{4}^{\prime}\right\} \\
+\left\{u_{12}+a_{1}^{\prime \prime}\right\}-\left\{u_{12}+1+a_{2}^{\prime \prime}\right\}-\left\{u_{12}+2+a_{3}^{\prime \prime}\right\}-\left\{u_{12}+3+a_{4}^{\prime \prime}\right\}=0, \\
+\left\{u_{12}-8+a_{1}\right\}-\left\{u_{12}-7+a_{2}\right\}-\left\{u_{12}-6+a_{3}\right\}-\left\{u_{12}-5+a_{4}\right\} \\
-\left\{u_{12}-4+a_{1}^{\prime}\right\}+\left\{u_{12}-3+a_{2}^{\prime}\right\}-\left\{u_{12}-2+a_{3}^{\prime}\right\}+\left\{u_{12}-1+a_{4}^{\prime}\right\} \\
+\left\{u_{12}+a_{1}^{\prime \prime}\right\}+\left\{u_{12}+1+a_{2}^{\prime \prime}\right\}+\left\{u_{12}+2+a_{3}^{\prime \prime}\right\}-\left\{u_{12}+3+a_{4}^{\prime \prime}\right\}=0, \\
+\left\{u_{12}-8+a_{1}\right\}+\left\{u_{12}-7+a_{2}\right\}+\left\{u_{12}-6+a_{3}\right\}-\left\{u_{12}-5+a_{4}\right\} \\
+\left\{u_{12}-4+a_{1}^{\prime}\right\}-\left\{u_{12}-3+a_{2}^{\prime}\right\}-\left\{u_{12}-2+a_{3}^{\prime}\right\}-\left\{u_{12}-1+a_{4}^{\prime}\right\} \\
-\left\{u_{12}+a_{1}^{\prime \prime}\right\}+\left\{u_{12}+1+a_{2}^{\prime \prime}\right\}-\left\{u_{12}+2+a_{3}^{\prime \prime}\right\}+\left\{u_{12}+3+a_{4}^{\prime \prime}\right\}=0 .
\end{array}\right.
$$

The second and third equations in IV are derived from the last set in the $(12 n+1)$-th and $(12 n+2)$-th term, respectively. The $(12 n+3)$-th, 
$(12 n+6)$-th, and $(12 n+9)$-th terms yield the same equation in IV as the $(12 n)$-th term; the $(12 n+4)$-th, $(12 n+7)$-th, $(12 n+10)$-th terms the same as the $(12 n+1)$-th term; the $(12 n+5)$-th, $(12 n+8)$-th, $(12 n+11)$-th the same as the $(12 n+2)$-th term.

The equations II, when simplified and by the substitution of $v_{12 p}$ for $v_{12}$ made to apply to other sets in the terms of the product, are

$$
\left\{\begin{aligned}
-\left\{v_{12 p}-8+\beta_{2}\right\} & +\left\{v_{12 p}-6+\beta_{1}^{\prime}\right\}+\left\{v_{12 p}-4+\beta_{3}^{\prime}\right\} \\
& +\left\{v_{12 p}-2+\beta_{2}^{\prime \prime}\right\}-\left\{v_{12 p}+\beta_{1}^{\prime \prime \prime}\right\}-\left\{v_{12 p}+2+\beta_{3}^{\prime \prime \prime}\right\}=0, \\
\left\{v_{12 p}-9+\beta_{1}\right\} & +\left\{v_{12 p}-7+\beta_{3}\right\}+\left\{v_{12 p}-5+\beta_{2}^{\prime}\right\} \\
- & \left\{v_{12 p}-3+\beta_{1}^{\prime \prime}\right\}-\left\{v_{12 p}-1+\beta_{3}^{\prime \prime}\right\}-\left\{v_{12 p}+1+\beta_{2}^{\prime \prime \prime}\right\}=0 .
\end{aligned}\right.
$$

The equations IV, when simplified, $u_{12 p}$ being written for $u_{12}$, become

$$
\text { (VI) }\left\{\begin{array}{l}
\left\{u_{12 p}-7+a_{2}\right\}+\left\{u_{12 p}-4+a_{1}^{\prime}\right\}-\left\{u_{12 p}-1+a_{4}^{\prime}\right\}-\left\{u_{12 p}+2+a_{3}^{\prime \prime}\right\}=0, \\
\left\{u_{12 p}-8+a_{1}\right\}-\left\{u_{12 p}-5+a_{4}\right\}-\left\{u_{12 p}-2+a_{3}^{\prime}\right\}+\left\{u_{12 p}+1+a_{2}^{\prime \prime}\right\}=0, \\
\left\{u_{12 p}-6+a_{3}\right\}-\left\{u_{12 p}-3+a_{2}^{\prime}\right\}-\left\{u_{12 p}+a_{1}^{\prime \prime}\right\}+\left\{u_{12 p}+3+a_{4}^{\prime \prime}\right\}=0 .
\end{array}\right.
$$

If we aim to assign to the Greek letters constant values, then, in every equation in $\mathrm{V}$ each fraction must admit of being associated singly with another fraction in that equation which is numerically equal to it, but of opposite sign. This relation is brought about by the following six equations:

$$
\text { (VII) } \begin{cases}\left\{v_{12 p}-9+\beta_{1}\right\}=\left\{v_{12 p}-3+\beta_{1}^{\prime \prime}\right\}, & \left\{v_{12 p}-6+\beta_{1}^{\prime}\right\}=\left\{v_{12 p}+\beta_{1}^{\prime \prime}\right\}, \\ \left\{v_{12 p}-8+\beta_{2}\right\}=\left\{v_{12 p}-2+\beta_{2}^{\prime \prime}\right\}, & \left\{v_{12 p}-5+\beta_{2}^{\prime}\right\}=\left\{v_{12 p}+1+\beta_{2}^{\prime \prime}\right\}, \\ \left\{v_{12 p}-7+\beta_{3}\right\}=\left\{v_{12 p}-1+\beta_{3}^{\prime \prime}\right\}, & \left\{v_{12 p}-4+\beta_{3}^{\prime}\right\}=\left\{v_{12 p}+2+\beta_{3}^{\prime \prime \prime}\right\} .\end{cases}
$$

The same considerations applied to equations VI yield the following six relations :

(VIII) $\begin{cases}\left\{u_{12 p}-8+a_{1}\right\}=\left\{u_{12 p}-2+a_{3}^{\prime}\right\}, & \left\{u_{12 p}-5+a_{4}\right\}=\left\{u_{12 p}+1+a_{2}^{\prime \prime}\right\}, \\ \left\{u_{12 p}-7+a_{2}\right\}=\left\{u_{12 p}-1+a_{4}^{\prime}\right\}, & \left\{u_{12 p}-4+a_{1}^{\prime}\right\}=\left\{u_{12 p}+2+a_{3}^{\prime \prime}\right\}, \\ \left\{u_{12 p}-6+a_{3}\right\}=\left\{u_{12 p}+a_{1}^{\prime \prime}\right\}, & \left\{u_{12 p}-3+a_{2}^{\prime}\right\}=\left\{u_{12 p}+3+a_{4}^{\prime \prime}\right\} .\end{cases}$

Applying relations VIII, one may write the series $\sum u_{m}$ thus, the parentheses being suppressed :

$$
\left\{\begin{aligned}
\sum u_{m} \equiv \sum_{p=1}^{p=\infty}\left(\left\{u_{12 p}-8+a_{1}\right\}+\left\{u_{12 p}-7+a_{2}\right\}-\left\{u_{12 p}-6+a_{3}\right\}\right. \\
-\left\{u_{12 p}-5+a_{4}\right\}+\left\{u_{12 p}-4+a_{1}^{\prime}\right\}+\left\{u_{12 p}-3+a_{2}^{\prime}\right\} \\
-\left\{u_{12 p}-8+a_{1}\right\}-\left\{u_{12 p}-7+a_{2}\right\}+\left\{u_{12 p}-6+a_{3}\right\} \\
\left.+\left\{u_{12 p}-5+a_{4}\right\}-\left\{u_{12 p}-4+a_{1}^{\prime}\right\}-\left\{u_{12 p}-3+a_{2}^{\prime}\right\}\right)
\end{aligned}\right.
$$


Applying relations VII, one may write the series $\sum v_{m}$ thus, the parentheses being suppressed :

$$
\left\{\begin{aligned}
\sum v_{m} \equiv \sum_{p=1}^{p=\infty} & \left(\left\{v_{12 p}-9+\beta_{1}\right\}+\left\{v_{12 p}-8+\beta_{2}\right\}-\left\{v_{12 p}-7+\beta_{3}\right\}\right. \\
& +\left\{v_{12 p}-6+\beta_{1}^{\prime}\right\}+\left\{v_{12 p}-5+\beta_{2}^{\prime}\right\}-\left\{v_{12 p}-4+\beta_{3}^{\prime}\right\} \\
& +\left\{v_{12 p}-9+\beta_{1}\right\}+\left\{v_{12 p}-8+\beta_{2}\right\}-\left\{v_{12 p}-7+\beta_{3}\right\} \\
& \left.+\left\{v_{12 p}-6+\beta_{1}^{\prime}\right\}+\left\{v_{12 p}-5+\beta_{2}^{\prime}\right\}-\left\{v_{12 p}-4+\beta_{3}^{\prime}\right\}\right) .
\end{aligned}\right.
$$

Series IX is conditionally convergent; series $\mathrm{X}$ divergent. In both series any constant positive values may be assigned to the Greek letters. The product of the two series is absolutely convergent. This may be shown by examining the $12 n$-th term and the eleven immediately succeeding terms of the product-series in the same way as we did the terms in the product of $S_{1}$ and $S_{2}$. We shall here write down the expression for the $(12 n+1)$-th term only, viz.,

$$
\begin{aligned}
& \sum_{p=1}^{p=n^{\prime}}(+\left\{u_{12 n-12 p}+16+a_{1}\right\}\left\{v_{12 p}-9+\beta_{1}\right\}-\left\{u_{12 n-12 p}+9+a_{2}^{\prime}\right\}\left\{v_{12 p}-8+\beta_{2}\right\} \\
&+\left\{u_{12 n-12 p}+8+a_{1}^{\prime}\right\}\left\{v_{12 p}-7+\beta_{3}\right\}+\left\{u_{12 n-12 p}+7+a_{4}\right\}\left\{v_{12 p}-6+\beta_{1}^{\prime}\right\} \\
&+\left\{u_{12 n-12 p}+6+a_{3}\right\}\left\{v_{12 p}-5+\beta_{2}^{\prime}\right\}+\left\{u_{12 n-12 p}+5+a_{2}\right\}\left\{v_{12 p}-4+\beta_{3}^{\prime}\right\} \\
&-\left\{u_{12 n-12 p}+4+a_{1}\right\}\left\{v_{12 p}-9+\beta_{1}\right\}+\left\{u_{12 n-12 p}+9+a_{2}^{\prime}\right\}\left\{v_{12 p}-8+\beta_{2}\right\} \\
&-\left\{u_{12 n-12 p}+8+a_{1}^{\prime}\right\}\left\{v_{12 p}-7+\beta_{3}\right\}-\left\{u_{12 n-12 p}+7+a_{4}\right\}\left\{v_{12 p}-6+\beta_{1}^{\prime}\right\} \\
&\left.-\left\{u_{12 n-12 p}+6+a_{3}\right\}\left\{v_{12 p}-5+\beta_{2}^{\prime}\right\}-\left\{u_{12 n-12 p}+5+a_{2}\right\}\left\{v_{12 p}-4+\beta_{3}^{\prime}\right\}\right) \\
&+\sum_{p=1}^{p=n^{\prime}}\left(+\left\{v_{12 n-12 p}+15+\beta_{1}\right\}\left\{u_{12 p}-8+a_{1}\right\}-\left\{v_{12 n-12 p}+8+\beta_{3}^{\prime}\right\}\left\{u_{12 p}-7+a_{2}\right\}\right. \\
&-\left\{v_{12 n-12 p}+7+\beta_{2}^{\prime}\right\}\left\{u_{12 p}-6+a_{3}\right\}-\left\{v_{12 n-12 p}+6+\beta_{1}^{\prime}\right\}\left\{u_{12 p}-5+a_{4}\right\} \\
&-\left\{v_{12 n-12 p}+5+\beta_{3}\right\}\left\{u_{12 p}-4+a_{1}^{\prime}\right\}+\left\{v_{12 n-12 p}+4+\beta_{2}\right\}\left\{u_{12 p}-3+a_{2}^{\prime}\right\} \\
&-\left\{v_{12 n-12 p}+3+\beta_{1}\right\}\left\{u_{12 p}-8+a_{1}\right\}+\left\{v_{12 n-12 p}+8+\beta_{3}^{\prime}\right\}\left\{u_{12 p}-7+a_{2}\right\} \\
&+\left\{v_{12 n-12 p}+7+\beta_{2}^{\prime}\right\}\left\{u_{12 p}-6+a_{3}\right\}+\left\{v_{12 n-12 p}+6+\beta_{1}^{\prime}\right\}\left\{u_{12 p}-5+a_{4}\right\} \\
&\left.+\left\{v_{12 n-12 p}+5+\beta_{3}\right\}\left\{u_{12 p}-4+a_{1}^{\prime}\right\}-\left\{v_{12 n-12 p}+4+\beta_{2}\right\}\left\{u_{12 p}-3+a_{2}^{\prime}\right\}\right)
\end{aligned}
$$

$-a$

$$
\begin{aligned}
= & -12 \sum_{p=1}^{p=n^{\prime}}\left\{v_{12 p}-9+\beta_{1}\right\}\left\{u_{12 n-12 p}+16+a_{1}\right\}\left\{u_{12 n-12 p}+4+a_{1}\right\} \\
& -12 \sum_{p=1}^{p=n^{\prime}}\left\{u_{12 p}-8+a_{1}\right\}\left\{v_{12 n-12 p}+15+\beta_{1}\right\}\left\{v_{12 n-12 p}+3+\beta_{1}\right\}-a,
\end{aligned}
$$

where $n^{\prime}=\frac{1}{2} n$ when $n$ is even, and $n^{\prime}=\frac{1}{2}(n+1)$ when $n$ is odd, and where $a$ is used in the same way as before. In this case $a$ consists of one constituent when $n$ is even, and of eleven constituents when $n$ is odd. By the very same reasoning as that applied in the series $S_{1}$ and $S_{2}$ it follows that the $(12 n+1)$-th 
term of the product of IX and X, for $n>m$, has a numerical value less than $1 / n(\log n)^{\theta}$, where $\theta>1$. The two factor-series IX and $\mathrm{X}$ differ from the pairs $S_{1}, S_{2}$ and $T_{1}, T_{2}$ in this-that (suppressing in each series each parenthesis which encloses a set) corresponding terms in IX and X need not have the same numerical values. However, the three pairs of factor-series have in common one property, to which allusion has not yet been made; viz., in one of the series of each pair, the sum of the terms in each set is identically zero, so that the sum of the series is itself zero.

Colorado Collegr, Colorado Springs. 